\title{
Serum insulin is associated with right ventricle function parameters and lung volumes in subjects free of cardiovascular disease
}

\author{
Ricarda von Krüchten ${ }^{1}$, Roberto Lorbeer ${ }^{2}$, Susanne Rospleszcz ${ }^{3,4}$, Corinna Storz ${ }^{5}$, Esther Askani ${ }^{1}$, \\ Charlotte Kulka', Wolfgang Rathmann ${ }^{6}$, Annette Peters ${ }^{3,4,7}$, Stefan Karrasch ${ }^{4,8,9}$, Fabian Bamberg ${ }^{1}$, \\ Christopher Schlett ${ }^{1}$ and Blerim Mujaj ${ }^{1}$
}

${ }^{1}$ Department of Diagnostic and Interventional Radiology, Universitäts Klinikum Freiburg, Faculty of Medicine, University of Freiburg, Freiburg, Germany, ${ }^{2}$ Department of Radiology, Ludwig-Maximilians-University Hospital, Munich, Germany, ${ }^{3}$ Chair of Epidemiology, Institute for Medical Information Processing, Biometry and Epidemiology, Medical Faculty, Ludwig-MaximiliansUniversität München, Munich, Germany, ${ }^{4}$ Institute of Epidemiology, Helmholtz Zentrum München, German Research Center for Environmental Health, Neuherberg, Germany, ${ }^{5}$ Department of Neuroradiology, Universitäts Klinikum Freiburg, Freiburg, Germany, ${ }^{6}$ German Diabetes Center, Institute for Biometrics and Epidemiology, Düsseldorf, Germany, ${ }^{7}$ German Center for Diabetes Research (DZD), partner site Neuherberg, Neuherberg, Germany, ${ }^{8}$ Institute and Outpatient Clinic for Occupational, Social and Environmental Medicine, Inner City Clinic, University Hospital of Munich, Ludwig-Maximilians-Universität, Munich, Germany, and ${ }^{9} \mathrm{Comprehensive} \mathrm{Pneumology} \mathrm{Center} \mathrm{Munich}$ (CPC-M), Member of the German Center for Lung Research (DZL), Munich/Neuherberg, Germany

Correspondence should be addressed to B Mujaj Email blerim.mujaj@uniklinikfreiburg.de

\begin{abstract}
Background: Diabetes mellitus is an established risk factor for cardiovascular diseases. Even impaired levels of glucose and insulin might harm organ function prior to diabetes onset. Whether serum glucose or insulin plays a direct role in cardiac dysfunction or lung volume reduction remains unclear. The aim was to investigate the relationship between glucose and insulin with the right ventricle and lung volumes within KORA-MRI FF4 study.

Methods: From the KORA-MRI FF4 cohort study 337 subjects (mean age $55.7 \pm 9.1$ years; $43 \%$ women) underwent a whole-body 3T MRI scan. Cardiac parameters derived from a cine-steady-state free precession sequence using cvi42. MRI-based lung volumes derived semi-automatically using an in-house algorithm. Fasting serum glucose, fasting insulin levels, and HOMA index were calculated in all study subjects. Linear regression analyses were performed to assess the relationships between glucose and insulin levels with right ventricle volumes and lung volumes adjusted for age, sex, BMI, and cardiovascular risk factors.

Results: In univariate and multivariate-adjusted models, high serum insulin was inversely associated with end-diastolic volume ( $\beta=-12.43, P<0.001)$, end-systolic volume $(\beta=-7.12, P<0.001)$, stroke volume $(\beta=-5.32, P<0.001)$, but not with ejection fraction. The association remained significant after additional adjustment for lung volumes. Similarly, serum insulin was inversely associated with lung volume $(\beta=-0.15, P=0.04)$. Sensitivity analysis confirmed results after excluding subjects with known diabetes.

Conclusions: Serum insulin was inversely associated with right ventricle function and lung volumes in subjects from the general population free of cardiovascular disease, suggesting that increased insulin levels may contribute to subclinical cardiopulmonary circulation impairment.
\end{abstract}




\section{Introduction}

Impaired glucose and insulin levels represent two major pathophysiological features of type 2 diabetes mellitus that may occur prior to diabetes disease onset $(1,2)$. Type 2 diabetes mellitus is an established risk factor for cardiovascular diseases (CVD) (3), and dysregulated levels of glucose and insulin have been implicated in the etiology of CVD in general (4). Moreover, under conditions of diabetes mellitus, subclinical cardiopulmonary dysfunction might aggravate $(5,6)$. In this context, the right ventricle and lungs constitute the main axis of the pulmonary circulation and their functional impairment reflects into cardiac output and may lead to pulmonary hypertension, especially among the elderly population.

Cardiac right ventricle (RV) function in subjects with prediabetes and type 2 diabetes within the Cooperative Health Research in the Region of Augsburg (KORA) cohort study was assessed, and the study reported an impaired right ventricle function in subjects with prediabetes and diabetes compared to controls (7). However, disruptions of levels may occur much earlier before diabetes onset (2) and the main driver of glucose metabolism is insulin (8). In addition, previous studies reported an association between insulin resistance and cardiac left ventricle (LV) dysfunction, particularly worsening of diastolic function $(9,10,11)$. The FLEMENGHO (Flemish Study on Environment, Genes, and Health Outcomes) study has shown that higher levels of insulin at baseline and follow-up were associated with the decrease of left ventricular systolic and diastolic function and an increase in left ventricular mass index (12). When matching the population-based cohort studies Study of Health in Pomerania (SHIP) and KORA, a relation between higher glucose and/or insulin levels and greater arterial stiffness, smaller LV chamber size and higher LV thickness with resultant LV concentric remodeling and lower LV stroke volume has been shown (13). The RV function closely related to the pulmonary circulation and lung volumes may represent important parameters for reciprocal function or dysfunction. Nevertheless, research on the RV function and pulmonary circulation, in general, remains underrepresented (14), especially in the context of the role of glucose and/or insulin. To date, no study assessed the relationship between glucose and insulin, RV function and/or lung volumes, and whole-body MRI technology enables reliable assessment of both, cardiac function and pulmonary volumes simultaneously during a single radiation-free MR scan examination (15).
Therefore, this study aimed to investigate the relationship between glucose and insulin with MRIderived RV and lung volumes in a population-based cohort study without known cardiovascular diseases.

\section{Methods}

\section{Study population}

The KORA S4 is a prospective population-based cohort study in the region of Augsburg, Germany (16). Subjects aged between 25 and 74 years, residents in the Augsburg region, were recruited in the study, and participants were examined between June 2013 and September 2014 at the KORA study center. A whole-body MRI scan (3 Tesla) was incorporated in the follow-up KORA-MRI FF4 study (17). Subjects were selected for an MRI scan if they met the following inclusion criteria: informed study consent, and qualification of being in the prediabetes, diabetes, or control group. The following exclusion criteria were applied: age $>74$ years; participants with a known history of coronary artery disease, myocardial infarction, peripheral artery disease, stroke, and/or unavailable oral glucose test, pregnancy, poor overall health condition or other physical limitations. Subjects with contraindications to MRI scan, such as known gadolinium contrast allergy, cardiac stents, cardiac pacemaker or implantable defibrillator, implanted metal parts, breast-feeding women, subjects with claustrophobia, and subjects with impaired renal function were excluded. From a total of participants with an MRI scan $(n=400)$ subjects with incomplete information on glucose $(n=2)$, insulin $(n=3)$, or HOMA index $(n=30)$ and incomplete visualization of the right ventricle $(n=28)$ were excluded. Hence, 337 subjects were included in the analysis (Fig. 1). The KORA-MRI FF4 study was approved by the Institutional Research Ethics Board of the Medical Faculty of Ludwig-Maximilian University Munich and compiled with Helsinki declaration on human research (18). All participants gave written informed consent.

\section{Whole-body MR imaging}

Whole-body MRI scans were performed with a 3-Tesla MRI system (Magnetom Skyra, Siemens AG, Healthcare Sector, Erlangen, Germany) (17) using an 18-channel body surface coil and a table-mounted spine matrix coil. The protocol comprised sequences covering the entire body (from neck to below hip) for tissue/organ quantification, as well as for particular organs, for example, brain, carotid 


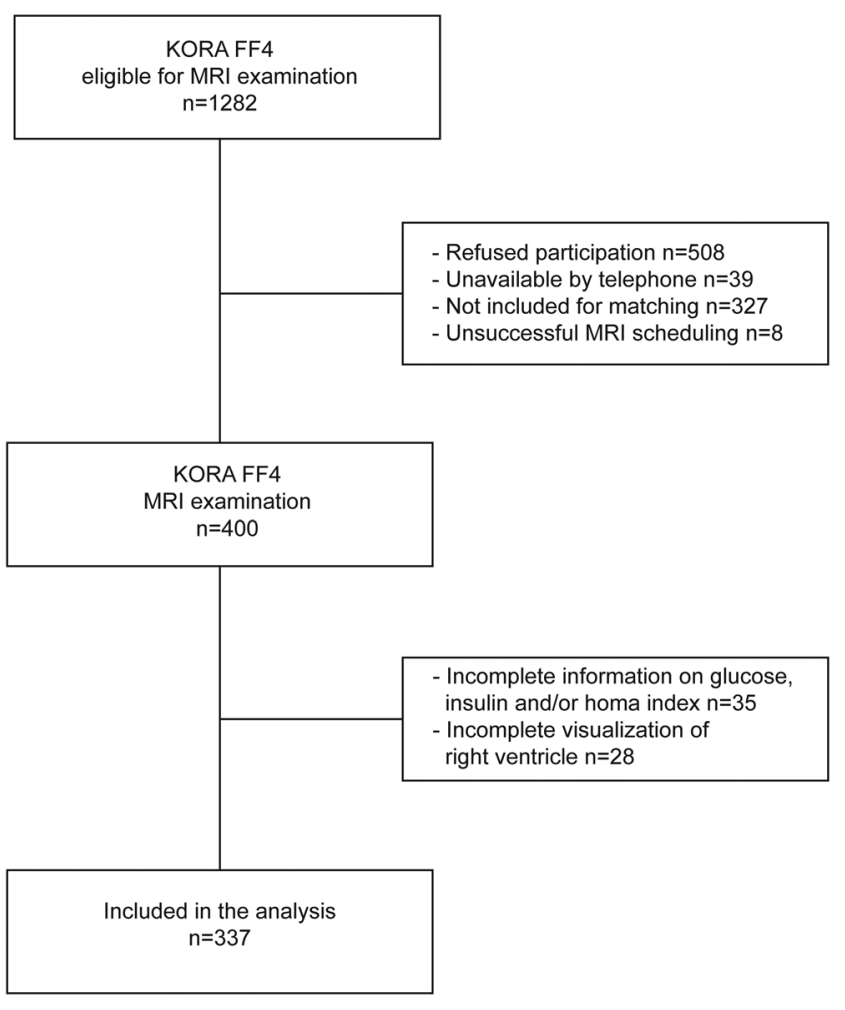

Figure 1

Flowchart of the study.

arteries, or fat compartments. For analysis of the lung, a 2-point DIXON T1 sequence was used in submaximal inspiration breath-hold and an acquisition time of $15 \mathrm{~s}$. Slice thickness was $3 \mathrm{~mm}$, coronal acquired, including a field of view (FOV) of $488 \mathrm{~mm} \times 716 \mathrm{~mm}$, a matrix of $256 \times 256$, a repetition time (TR) of $4.06 \mathrm{~ms}$, and an echo time (TE) of $1.26 \mathrm{~ms}$. For analysis of the heart, the cinesteady-state free precession sequence was acquired in a short-axis view with 10 layers and 25 phases. Slice thickness was $8 \mathrm{~mm}$, including a FOV of $297 \mathrm{~mm} \times 360 \mathrm{~mm}$, a matrix of $240 \times 160$, a TR of $29.97 \mathrm{~ms}$, a TE of $1.46 \mathrm{~ms}$, and a flip angle of $62^{\circ}$.

\section{MR-image analysis for cardiac volume}

RV function was evaluated using commercially available $\mathrm{cvi}^{42}$ software (version 4.1.5(190)); Circle Cardiovascular Imaging Inc. (Calgary, Alberta, Canada) by two independent readers. After manually segmenting the lumen of the RV in the end-systole and end-diastole in each layer from the cardiac apex to the pulmonary valve (19), the software calculated automatically the corresponding volumes. The difference between the end-systolic and end-diastolic volumes comprises the parameters for stroke volume and ejection fraction, according to defined normal values for cardiovascular magnetic resonance in adults and children values proposed by a recent publication (20).

\section{MR image analysis for lung volume}

An algorithm was used for the automatic procession of the MR data lung volumes as described previously in detail $(15,21)$. The algorithm consisted of the following segmentation steps: correction of intensity inhomogeneities, pre-extraction of a coarse region of interest containing the airways, segmentation of the bilateral lung and trachea regions, trachea extraction, and lung separation (right and left lung), and lung region refinement. Pulmonary blood vessels outside the mediastinal contours were included in the lung region (15). The whole MRI scan set after the automated processing was visually checked by an independent reader, unaware of the clinical information, and high-quality outputs of the framework have been verified (15).

\section{Assessment of serum glucose and insulin}

After overnight fasting venous blood was taken from all subjects at the study center. Serum fasting glucose (FG) was sampled, and $75 \mathrm{~g}$ of anhydrous glucose (Dextro OGT; Boehringer Mannheim, Mannheim, Germany) was given to participants without a known diagnosis of type 2 diabetes or taking glucose-lowering agents. Serum FG and $2 \mathrm{~h}$ post-load glucose levels (oral glucose tolerance test; OGTT) were measured using an enzymatic colorimetric method (Dimension Vista 1500, Siemens Healthcare Diagnostics, Eschborn, Germany or Cobas c702, Roche Diagnostics GmbH, Mannheim, Germany). Serum fasting insulin (FI) levels were determined from blood samples using serum FI and $2 \mathrm{~h}$ post-load insulin values were measured by a solid-phase enzyme-labeled chemiluminescent immunometric assay (Immulite 2000 Xpi, Siemens Healthcare Diagnostics) or by an electrochemiluminescence immunoassay (Cobas e 602, Roche Diagnostics $\mathrm{GmbH}$,). The homeostasis model assessment-insulin resistance index (HOMA-IR) was calculated using the formula: (fasting glucose $(\mathrm{mmol} / \mathrm{L}) \times$ fasting insulin $(\mu \mathrm{U} / \mathrm{L}) / 22.5)(22)$.

\section{Other risk factors}

Information on risk factors was obtained through physical examination, interview, and blood sampling. BMI and body surface area (BSA) were calculated, smoking 
status, alcohol use (g/day), and glucose-lowering, antihypertensive, lipid-lowering medication were assessed by questionnaire. Diabetes state was defined according to the WHO criteria as prediabetes (Impaired glucose tolerance, IGT: normal fasting glucose concentration and a 2-h serum OGTT glucose concentration between 140 and $200 \mathrm{mg} / \mathrm{dL}$; and/or an impaired fasting glucose concentration, as defined by fasting glucose levels between 110 and $125 \mathrm{mg} / \mathrm{dL}$, and a normal 2-h serum glucose concentration), and diabetes (2-h serum glucose concentration as determined by OGTT that was $>200 \mathrm{mg} /$ $\mathrm{dL}$ and/or a fasting glucose level that was $>125 \mathrm{mg} / \mathrm{dL}$ ) (23). Moreover, hypertension was defined as systolic blood pressure $>140 \mathrm{mmHg}$, diastolic blood pressure $>90 \mathrm{mmHg}$, or receiving current antihypertensive treatment. Glycated hemoglobin (HbA1c) was analyzed in hemolyzed whole blood using the cation-exchange high performance liquid chromatographic, photometric VARIANT II TURBO HbA1c Kit-2.0 assay on a VARIANT II TURBO Hemoglobin Testing System (Bio-Rad Laboratories Inc.). Total serum cholesterol and serum creatinine concentrations were analyzed using an enzymatic colorimetric method (Dimension Vista 1500, Siemens Healthcare Diagnostics, or Cobas c702, Roche Diagnostics GmbH). The estimated glomerular filtration rate (eGFR) was calculated based on creatinine/cystatin $\mathrm{C}$ or combination according to a standardized formula (24).

\section{Statistical analysis}

The distribution of study population characteristics was described by using mean and s.D., median (interquartile ranges (IQRs), or percentages for continuous and categorical variables, respectively. A natural logarithmic transformation was performed to normalize the distribution of insulin and HOMA index. We used a two-step approach to investigate the association between glucose, insulin, and HOMA index with cardiac parameters and lung volumes respectively. First, using linear regression models we investigated the association between fasting glucose, insulin, and HOMA index with cardiac parameters and lung volumes. In model 1, we adjusted for sex and age. Model 2 adjusted for smoking, alcohol use, BMI, systolic and diastolic blood pressure, total cholesterol, and eGFR. Model 3 was additionally adjusted for glycated hemoglobin (HbA1c), antihypertensive medication, lipid-lowering medication, and glucose, or insulin. In model 4 we addressed the potential confounding effect of the right ventricle function into lung function or vice versa and adjusted for right ventricle parameters or lung volumes, respectively. Second, a sensitivity adjustment was performed for all parameter associations in subjects without diabetes. All analyses were performed using Stata (Stata 16.1 Corporation).

\section{Results}

Table 1 summarizes the characteristics of the study population. The mean age of the population was $55.7 \pm 9.1$ years and $43 \%$ of subjects were women. Among the subjects, $21.4 \%$ were current smokers, and mean alcohol use was $18.3 \pm 22.3 \mathrm{~g} /$ day. Hypertension was present in $31.2 \%$ of subjects and only $5.3 \%$ of subjects had a diagnosis of diabetes mellitus. In all subjects, the mean (s.D.) fasting glucose was $5.53 \pm 0.69 \mathrm{mmol} / \mathrm{L}$, and median (IQR) fasting insulin was 56 (38-87) pmol/L.

\section{Association between serum insulin and right ventricle}

The association between serum insulin, HOMA index, and right ventricle is provided in Table 2 . In a model adjusted for sex and age, we found an inverse association between insulin and RV end-diastolic volume (per s.D. increase in insulin level: $\beta=-6.31, P=0.001$ ), end-systolic volume (per s.D. increase in insulin level: $\beta=-3.90, P=0.002$ ) and stroke volume (per S.D. increase in insulin level: $\beta=-2.43$, $P=0.017)$, but not with ejection fraction. The association remained significant after further adjustment for other risk factors in model 2 and 3 . When additionally adjusting for lung volumes in model 4 , the association remained significant for RV end-diastolic volume (per s.D. increase in insulin level: $\beta=-12.85, P \leq 0.001$ ), end-systolic volume (per s.D. increase in insulin level: $\beta=-7.26, P<0.001$ ) and stroke volume (per s.D. increase in insulin level: $\beta=-5.59$, $P<0.001$ ) (Fig. 2 and Table 2). Considering the HOMA index, we found similar association estimates for RV parameters and lung volumes in magnitude and direction to serum insulin (Table 2). Moreover, sensitivity analysis confirmed the results when restricting the analyses on subjects without a history of diabetes (Supplementary Table 1, see section on supplementary materials given at the end of this article). We found no association between glucose with any parameter of the right ventricle function (Table 2).

\section{Association between serum insulin and lung volume}

The inverse association was observed between insulin and lung volume in the model adjusted for sex and age 
Table 1 Study population characteristics. The subjects were stratified in tertiles (denoted as low, medium and high) of serum fasting insulin. The values represent mean \pm S.D., median (interquartile ranges) or frequency along with percentage.

\begin{tabular}{|c|c|c|c|c|c|}
\hline & \multicolumn{5}{|c|}{ Fasting insulin, pmol/L } \\
\hline & All & Low 13.9-43.2 & Medium 43.3-72.0 & High 72.1-288 & $P$ \\
\hline$n$ & 337 & 114 & 113 & 110 & \\
\hline Age, years & $55.7 \pm 9.1$ & $54.2( \pm 9.3)$ & $55.3( \pm 8.5)$ & $57.8( \pm 9.1)$ & 0.003 \\
\hline Women, \% & $145(43 \%)$ & $57(50 \%)$ & $49(43.4 \%)$ & $39(35.5 \%)$ & 0.028 \\
\hline $\mathrm{BMI}, \mathrm{kg} / \mathrm{m}^{2}$ & $27.9 \pm 4.7$ & $24.4( \pm 2.8)$ & $27.8( \pm 3.8)$ & $31.5( \pm 4.4)$ & $<0.001$ \\
\hline Body surface area, $\mathrm{m}^{2}$ & $1.95 \pm 0.21$ & $1.86( \pm 0.20)$ & $1.94( \pm 0.21)$ & $2.05( \pm 0.20)$ & $<0.001$ \\
\hline Smoking status & & & & & 0.861 \\
\hline Never, \% & $126(37.4 \%)$ & $45(39.5 \%)$ & $39(34.5 \%)$ & $42(38.2 \%)$ & \\
\hline Past, \% & $139(41.3 \%)$ & $42(36.8 \%)$ & $51(45.1 \%)$ & $46(41.8 \%)$ & \\
\hline Current, \% & $72(21.4 \%)$ & $27(23.7 \%)$ & $23(20.4 \%)$ & $22(20.0 \%)$ & \\
\hline Alcohol use, g/day & $18.3 \pm 22.3$ & $18.1( \pm 23.7)$ & $19.2( \pm 21.5)$ & $17.7( \pm 21.7)$ & 0.870 \\
\hline Diabetes status & & & & & $<0.001$ \\
\hline Normal, \% & $225(66.8 \%)$ & $98(86 \%)$ & $87(77 \%)$ & $40(36.4 \%)$ & \\
\hline Prediabetes, $\%$ & $94(27.9 \%)$ & $14(12.3 \%)$ & $24(21.2 \%)$ & $56(50.9 \%)$ & \\
\hline Diabetes, \% & $18(5.3 \%)$ & $2(1.8 \%)$ & $2(1.8 \%)$ & $14(12.7 \%)$ & \\
\hline $\mathrm{HbA} 1 \mathrm{c}, \%$ & $5.4 \pm 0.4$ & $5.4( \pm 0.3)$ & $5.4( \pm 0.3)$ & $5.6( \pm 0.5)$ & $<0.001$ \\
\hline Fasting glucose, $\mathrm{mmol} / \mathrm{L}$ & $5.53 \pm 0.69$ & $5.20( \pm 0.53)$ & $5.48( \pm 0.55)$ & $5.94( \pm 0.76)$ & $<0.001$ \\
\hline HOMA index & $2.2(1.4-3.5)$ & $1.2(0.9 ; 1.4)$ & $2.2(2 ; 2.6)$ & $4.0(3.5 ; 5.2)$ & $<0.001$ \\
\hline Hypertension, \% & $105(31.2 \%)$ & $19(16.7 \%)$ & $32(28.3 \%)$ & $54(49.1 \%)$ & $<0.001$ \\
\hline Systolic blood pressure, mm/Hg & $119.9 \pm 16.6$ & $113.7( \pm 14.7)$ & $120.1( \pm 14.1)$ & $126.2( \pm 18.6)$ & $<0.001$ \\
\hline Diastolic blood pressure, $\mathrm{mm} / \mathrm{Hg}$ & $75.4 \pm 9.9$ & $71.1( \pm 8.1)$ & $76.6( \pm 9.1)$ & $78.6( \pm 10.9)$ & $<0.001$ \\
\hline Antihypertensive medication, $\%$ & 77 (22.9\%) & $16(14.0 \%)$ & $21(18.6 \%)$ & $40(36.4 \%)$ & $<0.001$ \\
\hline Total cholesterol, mmol/L & $5.63 \pm 0.93$ & $5.49( \pm 0.86)$ & $5.74( \pm 0.85)$ & $5.68( \pm 1.05)$ & 0.285 \\
\hline Lipid lowering medication, \% & $29(8.6 \%)$ & $4(3.5 \%)$ & $11(9.7 \%)$ & $14(12.7 \%)$ & 0.014 \\
\hline eGFR, $\mathrm{mL} / \mathrm{min} / 1.73 \mathrm{~m}^{2}$ & $87.1 \pm 12.8$ & $88.7( \pm 12.5)$ & $87.9( \pm 12.6)$ & $84.8( \pm 13.1)$ & 0.030 \\
\hline \multicolumn{6}{|l|}{ Right ventricle } \\
\hline End-diastolic volume, $\mathrm{mL}$ & $166.9 \pm 39.7$ & $169.7( \pm 42.1)$ & $171.4( \pm 39.3)$ & $159.6( \pm 36.7)$ & 0.061 \\
\hline End-systolic volume, mL & $80.1 \pm 26.0$ & $82.3( \pm 28.7)$ & $82.0( \pm 25.1)$ & $75.7( \pm 23.7)$ & 0.123 \\
\hline Stroke volume, $\mathrm{mL}$ & $87.0 \pm 19.5$ & $87.5( \pm 19.6)$ & $89.4( \pm 19.1)$ & $83.9( \pm 19.5)$ & 0.171 \\
\hline Ejection fraction, $\%$ & $52.7 \pm 7.1$ & $52.3( \pm 7.4)$ & $52.7( \pm 6.4)$ & $53.1( \pm 7.6)$ & 0.429 \\
\hline Lung volume, L & $3.97( \pm 1.12)$ & $4.15( \pm 1.14)$ & $3.98( \pm 1.13)$ & $3.77( \pm 1.07)$ & 0.019 \\
\hline
\end{tabular}

$P$-value for trend.

eGFR, estimated glomerular filtration rate; HbA1c, glycated hemoglobin A1c.

(per s.D. increase in insulin level: $\beta=-0.23, P<0.001$ ). The association remained significant after further adjustment for risk factors in model 2 and 3. When adjusting for additional right ventricle function parameters in model 4 , the association remained significant (per S.D. increase in insulin level: $\beta=-0.18, P=0.017$ ) (Fig. 2 and Table 3). HOMA index provided similar effect estimates in magnitude and direction to serum insulin for the association with lung volumes. Furthermore, in sensitivity analysis, we found confirmatory results when concentrating the analyses on subjects without a history of diabetes (Supplementary Table 2). No association was found between serum glucose and lung volumes (Table 3).

\section{Discussion}

In this population-based cohort study, serum insulin was associated with reduced RV end-diastolic volume, end-systolic volume, stroke volume, but not with ejection fraction. Additionally, serum insulin was associated with reduced lung volumes.

Our findings suggest that serum insulin and insulin sensitivity may play an important role in the early stages of RV impairment and influences pulmonary circulation before diabetes disease onset. A recent KORA study found that in persons with diabetes or prediabetes RV function was reduced compared to healthy controls (7). Similarly, other studies focusing on LV function provided evidence on the role of an increased level of insulin and impaired LV function. In participants of SHIP and KORA studies, insulin was found to be related to a greater arterial stiffness, smaller LV volume, and higher LV thickness, resulting in LV concentric remodeling and lower LV stroke volume (13). Further, a study from FLEMENGHO found out that serum insulin was associated with longitudinal changes of LV (12). An independent association between the degree of insulin 


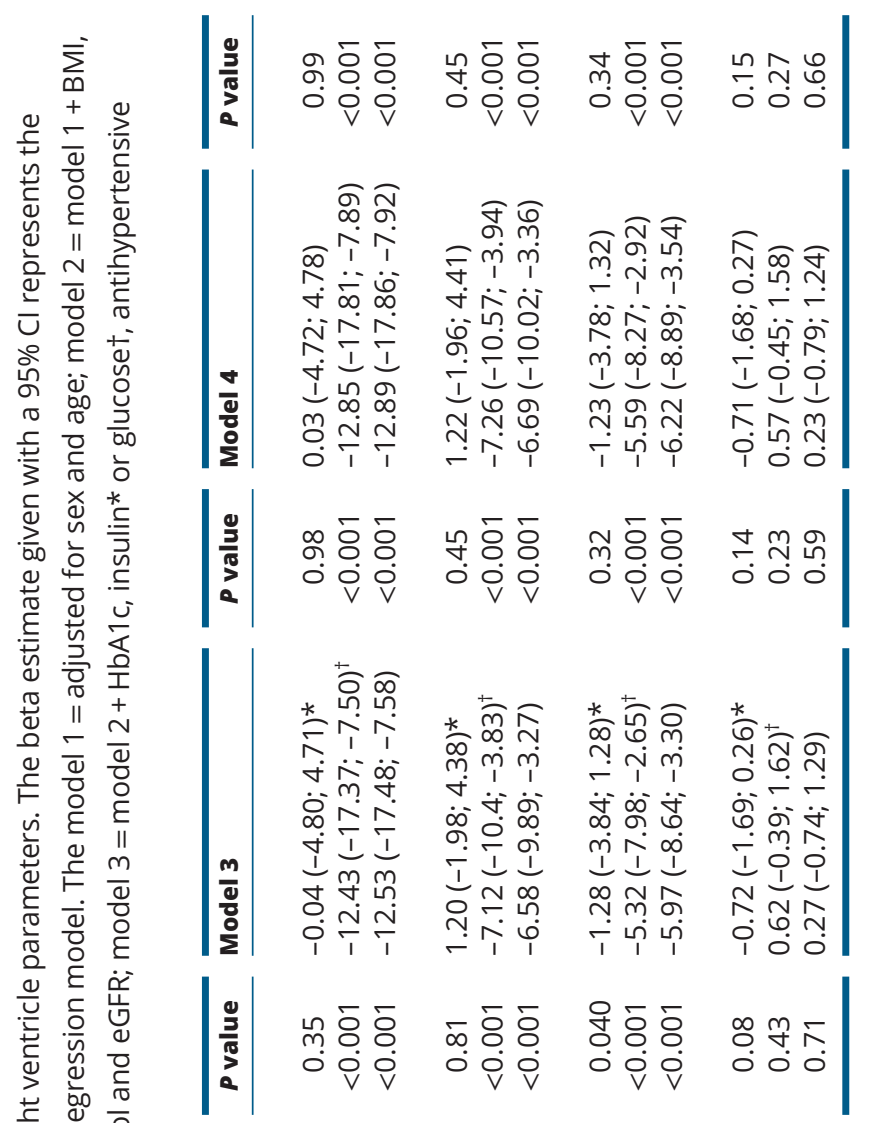

盾

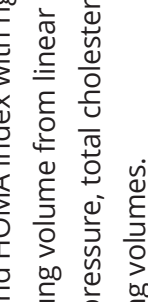

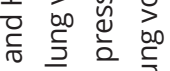

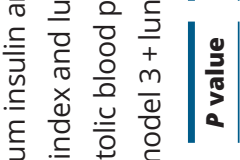

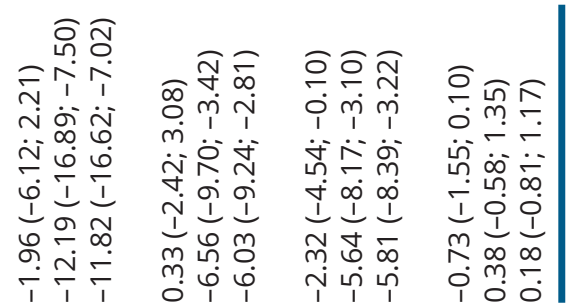

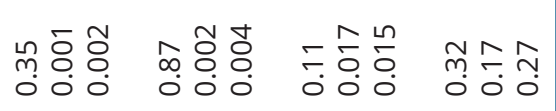

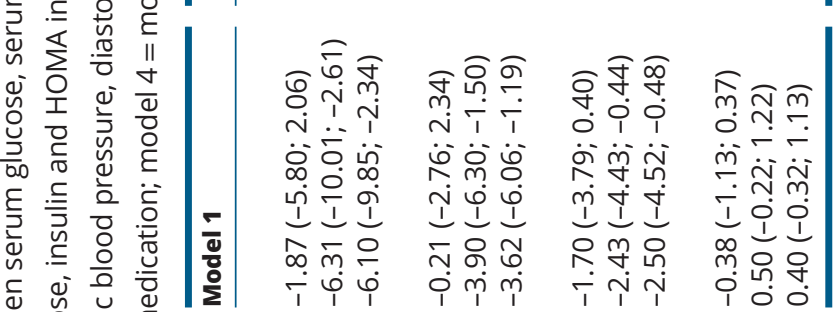



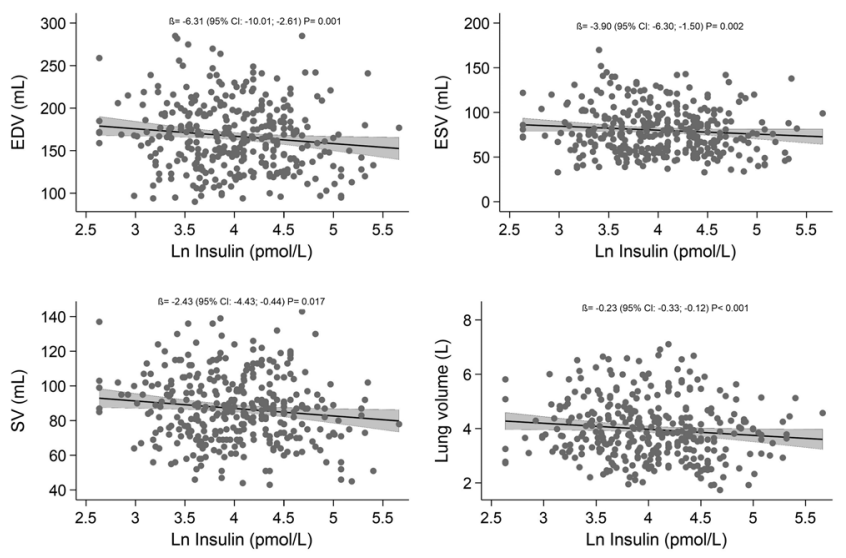

\section{Figure 2}

The relationship between serum insulin and right ventricle end-diastolic volume (EDV), end-systolic volume (ESV), stroke volume (SV), and lung volumes, scatter plots with regression line and $95 \% \mathrm{Cl}$.

resistance represented as HOMA index or hyperinsulinemia, and increased LV mass index, LV mass, and volume were observed in the MESA study (Multi-Ethnic Study of Atherosclerosis) (25). Furthermore, in the FRAMINGHAM heart study insulin resistance was associated with lower diastolic function, even before diabetes disease onset (9).

Given that our findings accounted for RV function, they are in line with previous studies involving the left ventricle and extend the evidence on the role of the insulin in the right heart also. The potential underlying pathophysiological mechanism may be a decreased glucose uptake by cardiomyocytes and consequently a lower glycolysis rate in the insulin-resistant state, with increased serum insulin beyond physiological levels (26, 27). Consequently, energy production from free fatty acids oxidation will increase and lipotoxicity may occur, leading to increased oxygen consumption, stress, and reduced cardiac contractility (28). Alternatively, serum insulin levels were associated with a higher presence of intraplaque hemorrhage in existing atherosclerotic plaque
(29), a plaque feature responsible for plaque progression in coronary arteries (30), leading to RV coronary ischemia (31). However, the link between the RV function and pulmonary circulation remains poorly understood (14). In this regard, we additionally assessed the role of insulin on lung volumes as a potential volumetric parameter of pulmonary pathology. Our findings build novel evidence on the role of insulin and reduced lung volumes. In this context, pulmonary vasculature seems to be affected by dysregulated levels of insulin. Abnormalities may result from the obliterative remodeling of pulmonary circulation which is characterized by occlusion of the lumen of medium-sized and small arteries in the lungs due to excessive cellular proliferation of vascular wall, and loss of microvasculature and capillaries due to elevation of serum insulin (32). This could be explained by insulin role on vascular endothelial growth factor which may induce further angiogenesis in pulmonary vessels (33), increase pulmonary vascular permeability in early stages of acute lung injury leading to loss of pulmonary volume (34), as well as impair the endothelial function of the pulmonary arteries leading to pulmonary hypertension (PH) (35). PH is a disease that predominantly affects distal pulmonary arteries with pathological changes including vasoconstriction, medial hypertrophy, intimal proliferation, and complex plexiform lesions (14). On this basis, we may hypothesize that through the combined interpretation of findings from $\mathrm{RV}$ and lung volumes insulin affects concomitantly RV function and pulmonary vasculature. Dysregulated serum insulin levels may occur even 15 years prior to diabetes disease onset (2), and this long-term effect may be sufficient for silent pathological changes in heart and lungs that at a later stage may be translated as overt PH disease in elderly subjects (36).

To date, there are no studies that assessed the role of insulin in RV and lung volumes as to the main axis of pulmonary circulation in disease-free subjects, and our findings may represent novel evidence in this matter and merit further investigation. Surprisingly, we did not

Table 3 Association between glucose, insulin and HOMA index with lung volume.

\begin{tabular}{|c|c|c|c|c|c|c|c|c|}
\hline \multirow[b]{2}{*}{ Per S.D. } & \multicolumn{8}{|c|}{ Lung volume } \\
\hline & Model 1 & $P$ value & Model 2 & $P$ value & Model 3 & $P$ value & Model 4 & $P$ value \\
\hline Glucose & $-0.10(-0.20 ; 0.01)$ & 0.08 & $-0.04(-0.15 ; 0.08)$ & 0.50 & $0.03(-0.11 ; 0.16)^{*}$ & 0.68 & $0.02(-0.12 ; 0.16)$ & 0.77 \\
\hline Insulin & $-0.23(-0.33 ;-0.12)$ & $<0.001$ & $-0.14(-0.28 ;-0.01)$ & 0.035 & $-0.15(-0.29 ;-0.01)^{\dagger}$ & 0.037 & $-0.18(-0.32 ;-0.03)$ & 0.017 \\
\hline HOMA index & $-0.22(-0.33 ;-0.12)$ & $<0.001$ & $-0.14(-0.28 ;-0.01)$ & 0.040 & $-0.13(-0.27 ; 0.01)$ & 0.06 & $-0.16(-0.31 ;-0.02)$ & 0.027 \\
\hline
\end{tabular}

The beta estimate given with a $95 \% \mathrm{CI}$ represents the estimate size between glucose, insulin and HOMA index and lung volume from linear regression model. The model 1 = adjusted for sex and age; model 2 = model 1 + BMI, smoking, alcohol use, systolic blood pressure, diastolic blood pressure, total cholesterol and eGFR; model $3=$ model $2+\mathrm{HbA} 1 \mathrm{c}$, insulin* or glucose ${ }^{\dagger}$, antihypertensive medication, lipid lowering medication; model $4=$ model $3+$ end-diastolic volume, end-systolic volume, stroke volume and ejection fraction. eGFR, estimated glomerular filtration rate; HbA1c, glycated hemoglobin A1c. 
find any role of glucose affecting RV function and lung volumes, despite previous evidence of serum glucose, and cardiovascular complications in general (37).

To our knowledge, our study is the first to assess the relation of serum insulin with RV cardiac function and lung volumes by combining both organs within a single MRI scan examination. The strengths of the study incorporate the implementation of advanced 3T whole-body MRI, which is superior to echocardiography in RV function assessment, in a population-based cohort without known cardiovascular disease. The MRI cardiac assessment and lung volumetric quantification was performed using advanced automatic and semi-automatic processes with a detailed protocol. Additionally, we performed a multi-level adjustment testing to confirm our results. Also, to rule out the possible overfitting issues and effect modification of the blood pressure, we accounted for testing with minimal adjustment. Given that high blood pressure is one of the main criteria for metabolic syndrome diagnosis (38) and a condition that is closely related to higher insulin levels, minimal adjustment model and fully adjusted models provided confirmatory results. However, a few limitations of the study warrant to be mentioned. The cross-sectional analysis limits to conclude causal inference, and our study may be regarded as hypothesis generation. Although we performed sensitivity analysis and excluded participants with known diabetes, a population-based cohort study consists of a rather small number of study participants.

\section{Conclusion}

Serum insulin was inversely associated with right ventricle function and lung volumes in subjects from the general population free of cardiovascular disease, suggesting that increased insulin levels may contribute to subclinical cardiopulmonary circulation impairment.

\section{Supplementary materials}

This is linked to the online version of the paper at https://doi.org/10.1530/ EJE-20-1010.

\section{Declaration of interest}

The authors declare that there is no conflict of interest that could be perceived as prejudicing the impartiality of this study.

\section{Funding}

The KORA study was initiated and financed by the Helmholtz Zentrum München - German Research Center for Environmental Health, which was funded by the German Federal Ministry of Education and Research (BMBF) and by the State of Bavaria. The study was funded by the German Research Foundation (DFG, Bonn, Germany; Project ID 245222810), the German Centre for Diabetes Research (DZD, Neuherberg Germany), and the German Centre for Cardiovascular Disease Research (DZHK, Berlin, Germany).

\section{Author contribution statement}

Study concept and design were performed by R V K, F B, C L S, and B M. Acquisition, analysis, or interpretation of data was performed by $R \vee K, R L$, $F B, C L S$, and B M. Drafting of the manuscript was performed by R V K, F B, $C L S$, and $B$ M. Critical revision of the manuscript for important intellectual content was performed by R V K, R L, S R, C S, E A, C K, W R, A P, S K, F B, $C L S$, and $B$ M. Statistical analysis was performed by $R$ L. Administrative, technical, or material support was performed by $R \vee K, F B, C L S$, and $B M$. Study supervision B M. All authors read and approved the final manuscript. $\mathrm{R} L$ had had full access to all the data in the study and takes responsibility for its integrity and the data analysis.

\section{References}

1 American Diabetes Association. Diagnosis and classification of diabetes mellitus. Diabetes Care 200932 (Supplement 1) S62-S67. (https://doi.org/10.2337/dc09-S062)

2 Nano J, Dhana K, Asllanaj E, Sijbrands E, Ikram MA, Dehghan A, Muka T \& Franco OH. Trajectories of BMI before diagnosis of Type 2 diabetes: the Rotterdam Study. Obesity 202028 1149-1156. (https:// doi.org/10.1002/oby.22802)

3 Mozaffarian D, Benjamin EJ, Go AS, Arnett DK, Blaha MJ, Cushman M, Das SR, de Ferranti S, Després J-P, Fullerton HJ et al. Heart disease and stroke statistics-2016 update. Circulation 2016133 38.e360. (https://doi.org/10.1161/CIR.0000000000000350)

4 Low Wang CC, Hess CN, Hiatt WR \& Goldfine AB. Clinical update: cardiovascular disease in diabetes mellitus: atherosclerotic cardiovascular disease and heart failure in type 2 diabetes mellitus - mechanisms, management, and clinical considerations. Circulation 2016133 2459-2502. (https://doi.org/10.1161/ CIRCULATIONAHA.116.022194)

5 Willson C, Watanabe M, Tsuji-Hosokawa A \& Makino A. Pulmonary vascular dysfunction in metabolic syndrome. Journal of Physiology 2019597 1121-1141. (https://doi.org/10.1113/JP275856)

6 Kenny HC \& Abel ED. Heart failure in type 2 diabetes mellitus. Circulation Research 2019124 121-141. (https://doi.org/10.1161/ CIRCRESAHA.118.311371)

7 Patscheider H, Lorbeer R, Auweter S, Schafnitzel A, Bayerl C, Curta A, Rathmann W, Heier M, Mesinger C, Peters A et al. Subclinical changes in MRI-determined right ventricular volumes and function in subjects with prediabetes and diabetes. European Radiology 201828 3105-3113. (https://doi.org/10.1007/s00330-017-5185-1)

8 Wallace TM, Levy JC \& Matthews DR. Use and abuse of HOMA modeling. Diabetes Care 200427 1487-1495. (https://doi. org/10.2337/diacare.27.6.1487)

9 Velagaleti RS, Gona P, Chuang ML, Salton CJ, Fox CS, Blease SJ, Yeon SB, Manning WJ \& O'Donnell CJ. Relations of insulin resistance and glycemic abnormalities to cardiovascular magnetic resonance measures of cardiac structure and function: the Framingham Heart Study. Circulation: Cardiovascular Imaging 20103 257-263. (https:// doi.org/10.1161/CIRCIMAGING.109.911438)

10 Demmer RT, Allison MA, Cai J, Kaplan RC, Desai AA, Hurwitz BE, Newman JC, Shah SJ, Swett K, Talavera GA et al. Association of impaired glucose regulation and insulin resistance with cardiac structure and function: results from ECHO-SOL (echocardiographic study of latinos). Circulation: Cardiovascular Imaging 20169 e005032. (https://doi.org/10.1161/CIRCIMAGING.116.005032) 
11 Fontes-Carvalho R, Ladeiras-Lopes R, Bettencourt P, Leite-Moreira A $\&$ Azevedo A. Diastolic dysfunction in the diabetic continuum: association with insulin resistance, metabolic syndrome and type 2 diabetes. Cardiovascular Diabetology 201514 4. (https://doi. org/10.1186/s12933-014-0168-x)

12 Cauwenberghs N, Knez J, Thijs L, Haddad F, Vanassche T, Yang WY, Wei FF, Staessen JA \& Kuznetsova T. Relation of insulin resistance to longitudinal changes in left ventricular structure and function in a general population. Journal of the American Heart Association $20187 \mathrm{e} 008315$. (https://doi.org/10.1161/ JAHA.117.008315)

13 Markus MRP, Rospleszcz S, Ittermann T, Baumeister SE, Schipf S, Siewert-Markus U, Lorbeer R, Storz C, Ptushkina V, Peters A et al. Glucose and insulin levels are associated with arterial stiffness and concentric remodeling of the heart. Cardiovascular Diabetology 2019 18 145. (https://doi.org/10.1186/s12933-019-0948-4)

14 Shah SJ. Pulmonary hypertension. JAMA 2012308 1366-1374. (https://doi.org/10.1001/jama.2012.12347)

15 Mueller J, Karrasch S, Lorbeer R, Ivanovska T, Pomschar A, Kunz WG, von Krüchten R, Peters A, Bamberg F, Schulz H et al. Automated MR-based lung volume segmentation in population-based wholebody MR imaging: correlation with clinical characteristics, pulmonary function testing and obstructive lung disease. European Radiology 2019 29 1595-1606. (https://doi.org/10.1007/s00330-018-5659-9)

16 Holle R, Happich M, Lowel H, Wichmann HE \& MONICA/KORA Study Group. KORA - a research platform for population based health research. Gesundheitswesen 200567 (Supplement 1) S19-S25. (https://doi.org/10.1055/s-2005-858235)

17 Bamberg F, Hetterich H, Rospleszcz S, Lorbeer R, Auweter SD, Schlett CL, Schafnitzel A, Bayerl C, Schindler A, Saam T et al. Subclinical disease burden as assessed by whole-body MRI in subjects with prediabetes, subjects with diabetes, and normal control subjects from the general population: the KORA-MRI Study. Diabetes 201766 158-169. (https://doi.org/10.2337/db16-0630)

18 World Medical Association. World Medical Association Declaration of Helsinki: ethical principles for medical research involving human subjects. JAMA 2013310 2191-2194. (https://doi.org/10.1001/ jama.2013.281053)

19 Schulz-Menger J, Bluemke DA, Bremerich J, Flamm SD, Fogel MA, Friedrich MG, Kim RJ, von Knobelsdorff-Brenkenhoff F, Kramer CM, Pennell DJ et al. Standardized image interpretation and post processing in cardiovascular magnetic resonance: Society for Cardiovascular Magnetic Resonance (SCMR) board of trustees task force on standardized post processing. Journal of Cardiovascular Magnetic Resonance 201315 35. (https://doi.org/10.1186/1532429X-15-35)

20 Kawel-Boehm N, Maceira A, Valsangiacomo-Buechel ER, VogelClaussen J, Turkbey EB, Williams R, Plein S, Tee M, Eng J \& Bluemke DA. Normal values for cardiovascular magnetic resonance in adults and children. Journal of Cardiovascular Magnetic Resonance 201517 29. (https://doi.org/10.1186/s12968-015-0111-7)

21 Ivanovska T, Hegenscheid K, Laqua R, Kühn JP, Gläser S, Ewert R, Hosten N, Puls R \& Völzke H. A fast and accurate automatic lung segmentation and volumetry method for MR data used in epidemiological studies. Computerized Medical Imaging and Graphics 201236 281-293. (https://doi.org/10.1016/j. compmedimag.2011.10.001)

22 Matthews DR, Hosker JP, Rudenski AS, Naylor BA, Treacher DF $\&$ Turner RC. Homeostasis model assessment: insulin resistance and beta-cell function from fasting plasma glucose and insulin concentrations in man. Diabetologia 198528 412-419. (https://doi. org/10.1007/BF00280883)

23 Definition and Diagnosis of Diabetes Mellitus and Intermediate Hyperglycemia. Geneva: WHO. (available at: https://www.who.int/ diabetes/publications/diagnosis_diabetes2006/en/)
24 Levey AS, Stevens LA, Schmid CH, Zhang YL, Castro AF, Feldman HI, Kusek JW, Eggers P,Van Lente F, Greene T et al. A new equation to estimate glomerular filtration rate. Annals of Internal Medicine 2009150 604-612. (https://doi.org/10.7326/0003-4819-150-9200905050-00006)

25 Shah RV, Abbasi SA, Heydari B, Rickers C, Jacobs Jr DR, Wang L, Kwong RY, Bluemke DA, Lima JAC \& Jerosch-Herold M. Insulin resistance, subclinical left ventricular remodeling, and the obesity paradox: MESA (Multi-Ethnic Study of Atherosclerosis). Journal of the American College of Cardiology 201361 1698-1706. (https://doi. org/10.1016/j.jacc.2013.01.053)

26 Szablewski L. Glucose transporters in healthy heart and in cardiac disease. International Journal of Cardiology 2017230 70-75. (https:// doi.org/10.1016/j.ijcard.2016.12.083)

27 Gibbs EM, Stock JL, McCoid SC, Stukenbrok HA, Pessin JE, Stevenson RW, Milici AJ \& McNeish JD. Glycemic improvement in diabetic $\mathrm{db} / \mathrm{db}$ mice by overexpression of the human insulinregulatable glucose transporter (GLUT4). Journal of Clinical Investigation 199595 1512-1518. (https://doi.org/10.1172/ JCI117823)

28 Witteles RM \& Fowler MB. Insulin-resistant cardiomyopathy clinical evidence, mechanisms, and treatment options. Journal of the American College of Cardiology 200851 93-102. (https://doi. org/10.1016/j.jacc.2007.10.021)

29 Mujaj B, Bos D, Kavousi M, van der Lugt A, Staessen JA, Franco OH \& Vernooij MW. Serum insulin levels are associated with vulnerable plaque components in the carotid artery: the Rotterdam Study. European Journal of Endocrinology 2020182 343-350. (https://doi. org/10.1530/EJE-19-0620)

30 Kolodgie FD, Gold HK, Burke AP, Fowler DR, Kruth HS, Weber DK, Farb A, Guerrero LJ, Hayase M, Kutys R et al. Intraplaque hemorrhage and progression of coronary atheroma. New England Journal of Medicine 2003349 2316-2325. (https://doi.org/10.1056/ NEJMoa035655)

31 Ford ES, Zhao G \& Li C. Pre-diabetes and the risk for cardiovascular disease: a systematic review of the evidence. Journal of the American College of Cardiology 201055 1310-1317. (https://doi.org/10.1016/j. jacc.2009.10.060)

32 Michelakis ED, Wilkins MR \& Rabinovitch M. Emerging concepts and translational priorities in pulmonary arterial hypertension. Circulation 2008118 1486-1495. (https://doi.org/10.1161/ CIRCULATIONAHA.106.673988)

33 He Z, Opland DM, Way KJ, Ueki K, Bodyak N, Kang PM, Izumo S, Kulkarni RN, Wang B, Liao R et al. Regulation of vascular endothelial growth factor expression and vascularization in the myocardium by insulin receptor and PI3K/Akt pathways in insulin resistance and ischemia. Arteriosclerosis, Thrombosis, and Vascular Biology 2006 26 787-793. (https://doi.org/10.1161/01.ATV.0000209500. $15801.4 \mathrm{e})$

34 Kaner RJ, Ladetto JV, Singh R, Fukuda N, Matthay MA \& Crystal RG. Lung overexpression of the vascular endothelial growth factor gene induces pulmonary edema. American Journal of Respiratory Cell and Molecular Biology 200022 657-664. (https://doi.org/10.1165/ ajrcmb.22.6.3779)

35 Partovian C, Adnot S, Raffestin B, Louzier V, Levame M, Mavier IM, Lemarchand P \& Eddahibi S. Adenovirus-mediated lung vascular endothelial growth factor overexpression protects against hypoxic pulmonary hypertension in rats. American Journal of Respiratory Cell and Molecular Biology 200023 762-771. (https://doi.org/10.1165/ ajrcmb.23.6.4106)

36 Gómez A, Bialostozky D, Zajarias A, Santos E, Palomar A, Martínez ML \& Sandoval J. Right ventricular ischemia in patients with primary pulmonary hypertension. Journal of the American College of Cardiology 200138 1137-1142. (https://doi.org/10.1016/s07351097(01)01496-6) 
37 Thrainsdottir IS, Aspelund T, Hardarson T, Malmberg K, Sigurdsson G, Thorgeirsson G, Gudnason V \& Rydén L. Glucose abnormalities and heart failure predict poor prognosis in the population-based Reykjavík Study. European Journal of Cardiovascular Prevention and Rehabilitation 200512 465-471. (https://doi. org/10.1097/01.hjr.0000173105.91356.4d)
38 Grundy SM, Brewer HB, Cleeman JI, Smith SC, Lenfant C, American Heart Association \& National Heart, Lung, and Blood Institute. Definition of metabolic syndrome: report of the National Heart, Lung, and Blood Institute/American Heart Association conference on scientific issues related to definition. Circulation 2004109 433-438. (https://doi.org/10.1161/01.CIR.0000111245.75752.C6)

Received 3 September 2020

Revised version received 10 November 2020

Accepted 17 November 2020 